\section{How good at neurology are you?}

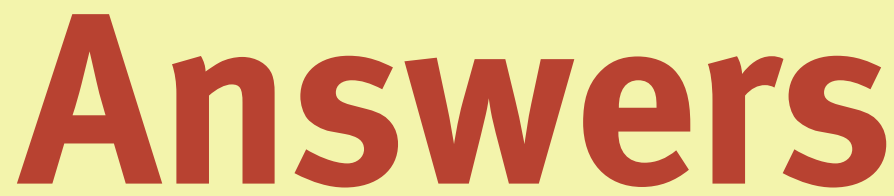

(See questions on $p, 46$ ) sort of tremor a rubral tremor, although thelesion site may vary from thalamus to midbrain, as in this case. Levodopa or a dopamineagonist may help.

\section{FURTHER READING}

Bain PG. (2002) The management of tremor. Journal of Neurology, N eurosurgery and Psychiatry, 72 (Suppl.1), 13-19.

1.

(a) (ii)

(b) (iii)

(c) (i)

(d) (iv) and perhaps(i)

(e) (v)

- M ild allergic reactions to IVIg are common. Anaphylactic reactions are rare and occur mostly in patients with an underlying IgA deficiency. Also, IVIg may occasionally lead to renal damage.

- Methotrexate causes interstitial fibrosis and should be avoided if there is already interstitial lung disease.

- Allopurinol inhibits xanthine oxidase, leading to decreased breakdown of azathioprine. Accumulation and eventual myelosuppression results, unless the dose of azathiorpineis appropriately lowered to $25 \%$ of usual, with particularly closemonitoring.

- Cyclosporine causes dose dependent nephrotoxicity, particularly in patients with preexisting renal failureand hypertension.

- Cyclophosphamide can cause a severe and potentially fatal haemorrhagic cystitis and so should beavoided if bladder inflammation is already present.

\section{Further reading:}

Chevrel, Goebels \& Hohlfeld. (2002) Myositis: diagnosis and management. Practical N eurology, 2, 4-11.

TheBritish National Formulary. http://www.bnf.org

2.

(c) TheM R showsatrophy of the right cerebral peduncle.

Holmes tremor is a slow $(<4.5 \mathrm{~Hz})$, irregular tremor, first described in detail by Gordon Holmes. It is present both at rest, with intention and often also on posture. It usually involves proximal and distal muscles. There is a variable delay from lesion to tremor onset, typically from 2 weeks to 2 years. Some people call this

\section{3.}

Huntington's disease. Patients with Huntington's disease have a tendency to progress from hyperkinetic movement disorders to parkinsonism and dystonia with time. It is well recognised that this process can be accelerated with neuroleptic treatment, sometimes to a catastrophic degree. Another condition which can present with psychiatric symptoms and an adverse reaction to neuroleptics is Lewy body dementia, but this is rarely familial to such a degree.

\section{FURTHER READING}

M ossJH, Stewart DE (1986) I atrogenic parkinsonism in Huntington's chorea. Can J Psychiatry, 31, 865-866.

4.

(a) sarcoid. This shows the circinate form of sarcoid, characterized by annular lesions with peripheral evolution and central clearing. The central area becomes depigmented and scarred. The lesions occur particularly on the face and neck.

(b) necrobiosis. Necrobiosis lipoidicum is due to a degeneration of connective tissue. Erythematous papules and nodules are seen in the pretibial area, with telangiectatic vessels over the papules. Over $50 \%$ of affected patients have diabetes.

5.

Lambert Eaton myasthenic syndrome (LEM S). A sneaky question. Following the first stimulation the patient contracted his muscle for 15 seconds, potentiating the response. He then relaxed, leading to a return of the baseline responseamplitude. Thisistheelectrophysiological counterpart of the post-exercise facilitation seen on reflex testing.

\section{FURTHER READING}

Dumitru D (1994) Electrodiagnostic M edicine. Hanley and Belfus Inc., Philadelphia. 